\title{
The Ji Chang hypothesis: Does perceptual learning alter perceived size?
}

\author{
Xinchi $\mathrm{Yu}$
}

School of Psychological and Cognitive Sciences, Peking University, China

An anecdote was noted in the book of Liezi (ca. 1045-221 BC), a Daoism classical literature: Ji Chang, a person who wanted to learn archery, hung a flea in front of his window, and looked at it all day. After around ten days, it became bigger; after three years, the flea seemed like a wheel to him. When he looked at other objects, they were as huge as mountains.

This anecdote could be seen as an early "testimony" of perceptual learning. More importantly, this story raises an interesting question for perceptual learning: are perceived size bigger after perceptual learning (here, visual perceptual learning)? For simplicity (and fun), let's refer to this question as the "Ji Chang hypothesis".

This is highly possible. Witt and Proffitt (2005) have demonstrated that, better batting in baseball correlates with bigger perceived size. However, there are two things about this research to keep in mind: one is the weak correlation coefficient $(r=0.29)$; another is that selecting circle size is probably not the best way to measure perceived size: it is possible that baseball players, just as Ji Chang, perceive every ball or circle as bigger, introducing some confounding factors. Similar criticism could also go with a similar research with golf players (Witt, Linkenauger, Bakdash and Proffitt, 2008). Likert scale would be a better idea.

Despite other possible explanations (as mentioned in the previous paragraph), let's assume that experienced players do perceive balls used in the games as bigger in size. This seems to be a support for the Ji Chang hypothesis. However, a classical argument could be that, you never know whether it is the case that only those who perceive bigger balls could become experienced professional players (or mostly so), or it is the case that perceptual learning (here, perceptual learning is embedded in years of training in this sport) alters the perceived size, similar to the famous case of London taxi drivers (Maguire, Woollett and Spiers, 2006).

Beside testing whether the Ji Chang hypothesis is true, there are also some other 
related questions to ask, for example, how perceptual learning changes the brain representation of the same object, and what is the relationship between brain representation pattern (for example, "sizes" in activation, namely activation level and activation area) and perceived size. It also brings us beyond the topic of "perceptual constancy", and provides us a scenario where "perceptual in-constancy" can be as intriguing.

\section{References}

Maguire, E. A., Woollett, K., \& Spiers, H. J. (2006). London taxi drivers and bus drivers: A structural MRI and neuropsychological analysis. Hippocampus, 16(12), 10911101

Witt, J. K., \& Proffitt, D. R. (2005). See the Ball, Hit the Ball Apparent Ball Size Is Correlated With Batting Average. Psychological Science, 16(12), 937-938.

Witt, J. K., Linkenauger, S. A., Bakdash, J. Z., \& Proffitt, D. R. (2008). Putting to a bigger hole: Golf performance relates to perceived size. Psychonomic Bulletin \& Review, 15(3), 581-585. 